\title{
Insulin resistance is associated with a metabolic profile of altered protein metabolism in Chinese and Asian-Indian men
}

\author{
E. S. Tai • M. L. S. Tan $•$ R. D. Stevens • Y. L. Low • \\ M. J. Muehlbauer • D. L. M. Goh • O. R. Ilkayeva • \\ B. R. Wenner • J. R. Bain • J. J. M. Lee • S. C. Lim • \\ C. M. Khoo • S. H. Shah • C. B. Newgard
}

Received: 24 August 2009 / Accepted: 24 November 2009/Published online: 15 January 2010

(C) Springer-Verlag 2010

\begin{abstract}
Aims/hypothesis Insulin resistance (IR) is associated with obesity, but can also develop in individuals with normal body weight. We employed comprehensive profiling methods to identify metabolic events associated with IR, while controlling for obesity.

Methods We selected 263 non-obese (BMI approximately $24 \mathrm{~kg} / \mathrm{m}^{2}$ ) Asian-Indian and Chinese men from a large cross-sectional study carried out in Singapore. Individuals taking medication for diabetes or hyperlipidaemia were excluded. Participants were separated into lower and upper tertiles of IR based on HOMA indices of $\leq 1.06$ or $\geq 1.93$,
\end{abstract}

Electronic supplementary material The online version of this article (doi:10.1007/s00125-009-1637-8) contains supplementary material, which is available to authorised users.

E. S. Tai $\cdot$ C. M. Khoo

Department of Medicine, Yong Loo Lin School of Medicine,

National University of Singapore,

Singapore, Republic of Singapore

E. S. Tai

Duke-National University of Singapore Graduate Medical School,

Singapore, Republic of Singapore

M. L. S. Tan

Singapore Health Services,

Singapore, Republic of Singapore

R. D. Stevens • M. J. Muehlbauer · O. R. Ilkayeva •

B. R. Wenner · J. R. Bain · S. H. Shah • C. B. Newgard ( $₫)$

Sarah W. Stedman Nutrition and Metabolism Center,

Duke Independence Park Facility,

4321 Medical Park Drive, Suite 200,

Durham, NC 27704, USA

e-mail: newga002@mc.duke.edu respectively. MS-based metabolic profiling of acylcarnitines, amino acids and organic acids was combined with hormonal and cytokine profiling in all participants.

Results After controlling for BMI, commonly accepted risk factors for IR, including circulating fatty acids and inflammatory cytokines, did not discriminate the upper and lower quartiles of insulin sensitivity in either AsianIndian or Chinese men. Instead, IR was correlated with increased levels of alanine, proline, valine, leucine/isoleucine, phenylalanine, tyrosine, glutamate/glutamine and ornithine, and a cluster of branched-chain and related amino acids identified by principal components analysis. These

Y. L. Low

Singapore Institute of Clinical Sciences,

Singapore, Republic of Singapore

D. L. M. Goh

Department of Pediatrics,

Yong Loo Lin School of Medicine,

National University of Singapore,

Singapore, Republic of Singapore

\section{J. J. M. Lee}

Department of Epidemiology and Public Health,

Yong Loo Lin School of Medicine,

National University of Singapore,

Singapore, Republic of Singapore

S. C. Lim

Alexandra Hospital,

Singapore, Republic of Singapore 
changes were not due to increased protein intake by individuals in the upper quartile of IR. Increased abdominal adiposity and leptin, and decreased adiponectin and IGF-binding protein 1 were also correlated with IR.

Conclusions/interpretation These findings demonstrate that perturbations in amino acid homeostasis, but not inflammatory markers or NEFAs, are associated with IR in individuals of relatively low body mass.

Keywords Body mass index - Branched-chain amino acids . Insulin resistance $\cdot$ Metabolomics $\cdot$ Protein metabolism

$\begin{array}{ll}\text { Abbreviations } \\ \text { BCAA } & \text { Branched-chain amino acid } \\ \text { IGFBP } & \text { IGF-I-binding protein } \\ \text { IR } & \text { Insulin resistance } \\ \text { PCA } & \text { Principal components analysis }\end{array}$

\section{Introduction}

Although obesity and insulin resistance (IR) are closely linked, BMI explains only $22 \%$ of the variance of IR in the general population [1]. Individuals with the metabolic syndrome who do not meet the criteria for central obesity can still be insulin resistant, and exhibit a similarly increased risk of coronary artery disease [1, 2]. Several studies have shown strong correlations between dysregulated lipid metabolism and IR, with some reports proposing impaired mitochondrial handling of lipids and consequent accumulation of intracellular lipid-derived metabolites, such as diacylglycerols and ceramides [3-5], whereas others implicate increased fatty acid flux and accumulation of incompletely oxidised lipid metabolites in the mitochondria [6-8]. Protein and amino acid metabolism are also dysregulated in obese and insulin-resistant states or type 2 diabetes [9-14], but the extent to which this contributes to IR in normal-weight individuals is incompletely understood. In addition to changes in circulating lipids and amino acids, obese and insulin-resistant individuals exhibit changes in a wide array of circulating hormones and inflammatory mediators [14], some of which may make a direct contribution to IR $[7,8]$.

We have recently used comprehensive metabolic profiling ('metabolomics') to identify multiple metabolites from different biochemical classes that differ in obese compared with lean humans from the south-eastern USA. Multivariate statistical analysis showed that the principal component (cluster of metabolites) most strongly associated with IR (as measured by HOMA index) was one comprising branchedchain amino acids (BCAAs) and related metabolites [14]. A subsequent cross-sectional study in sedentary and dyslipidaemic individuals of varying body weight confirmed a strong relationship between a similar BCAA-related metabolite cluster and the insulin sensitivity index [15]. The source of increased BCAAs in the blood of insulin-resistant individuals is unknown; contributing factors could include increased protein intake in obese individuals or changes in rates of protein turnover or amino acid catabolism. A mechanistic link between elevated BCAAs and IR was suggested in studies in rats, in which supplementation of a high-fat diet with BCAAs caused an increase in circulating BCAAs and their metabolites, and contributed to development of IR [14].

The foregoing summary highlights the need for deeper understanding of metabolic, hormonal and inflammatory factors contributing to the development of IR. A particular opportunity may reside in studies of development of IR in Asian populations. The epidemic of type 2 diabetes mellitus is occurring most rapidly in Asia [16], and Asians often develop IR at relatively low BMI [17, 18]. Singapore comprises three major ethnic groups (Chinese, Malaysian and Asian-Indian) living in a small geographical area. The country has become completely urbanised over the last 50 years, accompanied by a rapid increase in the prevalence of diabetes mellitus, from $4.7 \%$ in 1984 [19] to $>8 \%$ in 1998 [20]. Compared with Chinese, Asian-Indians in Singapore have a much higher prevalence of type 2 diabetes mellitus, and exhibit greater IR, pathologies not accounted for by differences in BMI [20, 21]. AsianIndians also have greater abdominal adiposity (as measured by waist circumference) [22], lower HDL-cholesterol and a higher risk of coronary artery disease, but lower BP than Chinese [23]. It is thus possible that differences in the phenotype associated with IR are related to differences in the causes of IR in the different ethnic groups, but this idea has never been explored in a systematic fashion.

In the current study, we have measured a wide array of metabolic, hormonal and inflammatory mediators in AsianIndian and Chinese individuals from Singapore with relatively normal body weight (mean BMI approximately $24 \mathrm{~kg} / \mathrm{m}^{2}$ ), separated into upper and lower tertiles of insulin sensitivity. By studying Singaporean residents, we minimised demographic and societal variables that might influence metabolic control and insulin sensitivity. With this approach, we sought to address the following key questions: (1) which of the elements of the metabolic profile associated with obesity is independently associated with IR and (2) when controlled for BMI, which metabolic features differ in the two ethnic groups living in Singapore? In this study population, we find a lack of association between IR and traditional IR biomarkers such as inflammatory mediators and fatty acids. IR is instead associated with changes in amino acid metabolism, visceral adiposity, the levels of two adipokines, leptin and adiponectin, and IGF-I-binding protein (IGFBP)-1. 


\section{Methods}

Participant recruitment and characteristics We invited 10,445 individuals from four previously conducted population-based cross-sectional surveys carried out in Singapore (1982-1998) [20, 22, 24-26] to participate in a repeat examination from 2004 to 2007. All studies were a random sample of Chinese, Malay and Asian-Indian individuals from the Singaporean population. Individuals who had died by the time of follow-up were excluded $(n=517)$. Also excluded were six individuals who had emigrated and 85 individuals who had errors in the records for the Identity Card number. All contactable individuals were invited to attend a health examination for additional tests and collection of biological specimens shortly after the home visit. A total of 7,742 participants completed the questionnaire, of whom 5,157 also attended the health examination.

Data on demographics and lifestyle factors, as well as medical history (including physician-diagnosed hypertension, diabetes mellitus and hyperlipidaemia) were collected using interviewer-administered questionnaires. Dietary intake was assessed using a semi-quantitative food frequency questionnaire, as described before [27-30].

For the health examination, participants were examined in the morning following a $10 \mathrm{~h}$ overnight fast. Weight was measured in light clothing using the same digital scale (SECA, model 782 2321009; Vogel and Halke, Hamburg, Germany) for all participants. Waist circumference was measured with a non-elastic tape at the mid-point between the costal margin and the iliac crest in the mid-axillary line. Hip circumference was measured at the level of the greater trochanter. Two readings of BP were taken from participants after a 5 min rest, using an automated BP monitor (Dinamap Pro100V2; Criticon, Norderstedt, Germany). Hypertension was defined as systolic BP $>140 \mathrm{mmHg}$ or diastolic BP $>90 \mathrm{mmHg}$ or history of hypertension or currently taking anti-hypertensive medications.

Participant selection for metabolic profiling We studied only men in order to reduce the potential confounding effects of sex as well as metabolic alterations occurring at different phases of the menstrual cycle. Furthermore, we chose to study only Chinese and Asian-Indian men, as these were the ethnic groups that show the greatest differences in IR in our population $[21,22]$. In order to examine the metabolic and hormonal correlates of IR as opposed to obesity, we first separated Asian-Indian and Chinese men into the upper and lower tertiles of IR, as measured by HOMA. These opposing tertiles corresponded to HOMA indices of $\leq 1.06$ and $\geq 1.93$. We next excluded all those individuals who were taking medications for diabetes or hyperlipidaemia, those who did not provide a urine specimen, and those with missing height and weight information. Of the 56 Asian-Indians with low
HOMA index, 30 had a BMI between 21 and $29 \mathrm{~kg} / \mathrm{m}^{2}$ (non-obese). For each of these individuals, we selected up to three individuals from the other three groups, with BMI within $0.3 \mathrm{~kg} / \mathrm{m}^{2}$. Of all the participants available we were able to identify 97 Chinese with low HOMA, 83 Chinese with high HOMA and 53 Asian-Indians with high HOMA with BMI within $0.3 \mathrm{~kg} / \mathrm{m}^{2}$ of an Asian-Indian individual with low HOMA, giving a total sample size of $263 \mathrm{men}$.

Measurement of insulin sensitivity Insulin sensitivity was measured by HOMA [31]. In what follows, we will refer to Asian-Indian or Chinese individuals in the upper tertile for HOMA interchangeably as 'high HOMA' or 'insulin resistant'.

Biochemical measurements Venous blood was drawn and collected in plain and fluoride oxalate tubes in the morning following a $10 \mathrm{~h}$ overnight fast. Some biochemical analyses on blood were carried out at the National University Hospital Referral Laboratory in Singapore. Serum total cholesterol, triacylglycerol and HDL-cholesterol were measured using an automated autoanalyser (ADVIA 2400; Bayer Diagnostics, Leverkusen, Germany). LDL-cholesterol levels were calculated using the Friedewald formula. Plasma glucose was also assayed using enzymatic methods (ADVIA 2400, Bayer Diagnostics) using blood collected in fluoride oxalate tubes.

All other metabolic, hormonal and inflammatory marker assays were performed at the Sarah W. Stedman Nutrition and Metabolism Center, Duke University. Commercial RIAs were used to measure leptin (Millipore, St Charles, MO, USA) and IGF-I (Beckman-Coulter, Webster, TX, USA). Searchlight chemiluminescent technology (Thermo Pierce-Endogen, Woburn, MA, USA) was used for measuring three IGFBPs (IGFBP1, IGFBP2, IGFBP3) using a custom multiplex assay. Adiponectin was measured via technology from Meso Scale Discovery (Gaithersburg, MD, USA), as was a nine plex cytokine panel for granulocyte-macrophage colony-stimulating factor, IFN- $\gamma$, IL-10, IL-12p70, IL-1 $\beta$, IL-2, IL-6, IL- 8 and TNF- $\alpha$. Total NEFAs, ketones and $\beta$-hydroxybutyrate (reagents from Wako, Richmond, VA, USA) and lactate (Roche, Indianapolis, IN, USA) were measured on a Hitachi 911 autoanalyser, as described previously [14].

Acylcarnitines and amino acids were analysed in plasma by tandem MS (MS/MS), and urine organic acids by GC/ MS, as described previously [14, 32-35]. All MS analyses employed stable-isotope dilution with internal standards from Isotec (St Louis, MO, USA), Cambridge Isotope Laboratories (Andover, MA, USA) and CDN Isotopes (Pointe-Claire, QC, Canada) [14, 33].

Ethics approval was obtained from two Institutional Review Boards (National University of Singapore and Singapore General Hospital). Informed consent was obtained before the conduct of the study. 
Statistical analyses Statistical analysis was carried out using STATA 8.0 (StataCorp LP; College Station, TX, USA and SAS v9.1.3, Cary, USA). Categorical variables were compared using the $\chi^{2}$ test. Continuous variables were compared using ANOVA. We first used an $F$ test to identify those variables for which at least one of the four groups showed a statistically significant difference from the others. Subsequently, the levels of metabolites in high- and low-HOMA groups were compared using one-way ANOVA with a Bonferroni multiple-comparison test, with and without adjustment for age and BMI. $p<0.05$ was taken as statistically significant.

We also employed principal components analysis (PCA) to reduce the large number of correlated variables into uncorrelated factors. PCA was constructed on the full set of study individuals using all measured metabolites and hormones, without prior knowledge of ethnicity or HOMA. Factors with an eigen value $\geq 1.0$ were identified and varimax rotation performed to produce interpretable factors. Metabolites with a factor load $\geq 0.4$ were reported as composing a factor. Scoring coefficients were constructed from the initial group and used to calculate factor scores for each individual (weighted sum of the standardised metabolites within that factor, weighted on the factor loading for each metabolite). Statistical analyses of these metabolite factors was similar as that for the individual metabolites, namely an $F$ test was first used to identify factors that were significantly different between groups, and subsequently factor levels in high- and low-HOMA groups were compared using ANOVA, with and without adjustment for age and BMI.

\section{Results}

Demographic and clinical characteristics of participants Because we measured a total of 100 separate analytes in this study, Tables 1, 2, 3 and 4 summarise only those analytes for which at least one of the four groups showed a statistically significant difference from the others with $p<$ 0.05 . Data for analytes that did not differ significantly among groups can be found in Electronic supplementary material (ESM) Tables 1 and 2. Table 1 presents demographic and clinical characteristics of the Chinese and Asian-Indian study participants, stratified by IR. Individuals from the top tertile of IR in both ethnic groups had HOMA indices three to four times higher than those from the lowest tertile. These differences were driven almost exclusively by elevations in fasting insulin levels in the high-HOMA groups. Despite our attempts to match for BMI (mean BMI values among the four groups were within a tight range of $23.89-24.95 \mathrm{~kg} / \mathrm{m}^{2}$ ), BMI was slightly higher in individuals in the high-HOMA groups. Hip circumference was identical between the groups, whereas waist circumference, and consequently WHR, varied significantly between high- and low-HOMA participants in both ethnic groups. The levels of HDL-cholesterol were significantly lower in both groups of Asian-Indian individuals than in Chinese individuals, and HDL-cholesterol levels were lower in the high- vs low-HOMA individuals within both ethnic groups, whereas LDL-cholesterol levels were not associated with HOMA tertile or ethnic origin. Triacylglycerol levels were clearly elevated in the highHOMA individuals in both the Chinese and Asian-Indian groups (Table 1), whereas NEFA and ketone levels did not differ (Table 3 and ESM Table 1). Finally, BP (both systolic and diastolic) was modestly higher in the high-HOMA vs low-HOMA individuals within both ethnic groups (Table 1).

Dietary intake and physical activity of participants Table 2 shows the dietary intake and physical activity in these individuals. As a proportion of total energy intake, Asian-Indians consumed less protein and more saturated fat than Chinese. However, no dietary differences were noted between highand low-HOMA individuals in either ethnic group, including no difference in protein intake. There was a trend towards less physical activity in individuals with high HOMA that was not statistically significant ( $p=1.0$ between high- and low-HOMA groups in both Chinese and Asian-Indians).

Blood and urinary metabolites in study participants Table 3 shows the concentrations of individual metabolites in the various groups. Chinese individuals with high HOMA had higher levels of several amino acids than Chinese individuals with low HOMA. These included alanine, proline, valine, leucine/isoleucine, phenylalanine, tyrosine, glutamate/glutamine and ornithine. With the exception of phenylalanine, all these differences remained statistically significant when adjusted for age and BMI. Associations in the same direction were observed in Asian-Indians but reached statistical significance only for alanine, tyrosine and glutamate/glutamine.

We also measured a group of 20 urinary organic acids in all of the participant groups (Table 3). Lactate and pyruvate levels were increased in urine in the high-HOMA individuals of both ethnic groups; these changes were accompanied by significant increases in blood lactate levels as a function of HOMA, again in both ethnic groups. Interestingly, urinary isobutyrylglycine was significantly lower in the high-HOMA vs low-HOMA group in Asian-Indians but not in Chinese, and isovalerylglycine showed a similar trend. Methylmalonate, methylsuccinate and hydroxymethylglutarate levels were all higher in urine of Asian-Indian compared with Chinese individuals, but did not change significantly with HOMA in either ethnic group. Finally, among 45 acylcarnitine species measured by MS/MS, C10:1 and C8:1-dicarboxyl were low in insulin-resistant 
Table 1 Demographic and clinical characteristics

\begin{tabular}{|c|c|c|c|c|c|}
\hline \multirow[t]{2}{*}{ Variable } & \multicolumn{2}{|l|}{ Chinese } & \multicolumn{2}{|l|}{ Asian-Indian } & \multirow[t]{2}{*}{$p$ value $^{\mathrm{a}}$} \\
\hline & $\begin{array}{l}\text { Low HOMA } \\
(n=97)\end{array}$ & $\begin{array}{l}\text { High HOMA } \\
(n=83)\end{array}$ & $\begin{array}{l}\text { Low HOMA } \\
(n=30)\end{array}$ & $\begin{array}{l}\text { High HOMA } \\
(n=53)\end{array}$ & \\
\hline \multicolumn{6}{|l|}{ Cardiovascular risk factors } \\
\hline Age (years) & $46.8 \pm 11.5$ & $51.4 \pm 11.4$ & $50.8 \pm 11.5$ & $49.5 \pm 7.1$ & 0.037 \\
\hline Height $(\mathrm{cm})$ & $169.4 \pm 5.7$ & $169.4 \pm 6.9$ & $172.8 \pm 6.6$ & $170.5 \pm 7.5$ & 0.067 \\
\hline Weight (kg) & $68.7 \pm 7.3$ & $70.5 \pm 8.6$ & $72.2 \pm 7.6$ & $72.7 \pm 8.9$ & 0.018 \\
\hline BMI $\left(\mathrm{kg} / \mathrm{m}^{2}\right)$ & $23.9 \pm 1.9$ & $24.5 \pm 2.1$ & $24.2 \pm 2.1$ & $25.0 \pm 2.1$ & 0.017 \\
\hline Waist (cm) & $86.7 \pm 6.3$ & $90.6 \pm 7.00$ & $88.4 \pm 12.4$ & $93.2 \pm 7.0$ & 0.000006 \\
\hline Hip (cm) & $99.5 \pm 5.3$ & $100.0 \pm 5.5$ & $99.1 \pm 12.7$ & $101.5 \pm 6.0$ & 0.299 \\
\hline WHR & $0.87 \pm 0.05$ & $0.91 \pm 0.05$ & $0.89 \pm 0.08$ & $0.92 \pm 0.05$ & 0.000002 \\
\hline Systolic BP (mmHg) & $129 \pm 13$ & $139 \pm 18$ & $126 \pm 16$ & $134 \pm 16$ & 0.0002 \\
\hline Diastolic BP (mmHg) & $80 \pm 10$ & $85 \pm 12$ & $80 \pm 10$ & $83 \pm 11$ & 0.034 \\
\hline Insulin (pmol/1) & $25.0 \pm 7.4$ & $90.1 \pm 42.3$ & $24.5 \pm 8.5$ & $102.5 \pm 80.4$ & $1.455 \times 10^{-28}$ \\
\hline Glucose $(\mathrm{mmol} / \mathrm{l})$ & $4.73 \pm 0.50$ & $5.29 \pm 0.97$ & $4.76 \pm 0.57$ & $5.83 \pm 2.33$ & 0.000002 \\
\hline Total cholesterol (mmol/l) & $5.17 \pm 0.86$ & $5.35 \pm 0.82$ & $5.09 \pm 0.90$ & $5.35 \pm 0.83$ & 0.292 \\
\hline Triacylglycerol (mmol/l) & $1.30 \pm 0.61$ & $1.85 \pm 0.96$ & $1.38 \pm 0.99$ & $1.95 \pm 1.22$ & 0.000015 \\
\hline Total cholesterol/HDL-cholesterol ratio & $4.24 \pm 1.02$ & $4.68 \pm 1.22$ & $4.98 \pm 1.53$ & $5.21 \pm 1.13$ & 0.000016 \\
\hline HDL-cholesterol (mmol/l) & $1.33 \pm 0.27$ & $1.26 \pm 0.24$ & $1.15 \pm 0.30$ & $1.11 \pm 0.20$ & 0.000006 \\
\hline LDL-cholesterol (mmol/l) & $3.25 \pm 0.74$ & $3.25 \pm 0.74$ & $3.29 \pm 0.76$ & $3.33 \pm 0.87$ & 0.924 \\
\hline HOMA & $0.75 \pm 0.22$ & $3.02 \pm 1.58$ & $0.74 \pm 0.24$ & $3.70 \pm 3.02$ & $1.222 \times 10^{-27}$ \\
\hline C-reactive protein $(\mathrm{mg} / \mathrm{l})$ & $3.63 \pm 16.07$ & $2.32 \pm 3.34$ & $1.85 \pm 2.76$ & $5.50 \pm 17.39$ & 0.4743 \\
\hline \multicolumn{6}{|l|}{ Smoking (\%) } \\
\hline Yes & 35.1 & 42.0 & 36.7 & 38.0 & \\
\hline No & 64.9 & 58.0 & 63.3 & 62.0 & 0.826 \\
\hline \multicolumn{6}{|l|}{ Hypertension (\%) } \\
\hline Yes & 32.0 & 55.4 & 33.3 & 28.3 & \\
\hline No & 68.0 & 44.6 & 66.7 & 71.7 & 0.002 \\
\hline \multicolumn{6}{|l|}{ Antihypertensive medication (\%) } \\
\hline Yes & 13.2 & 25.8 & 12.0 & 7.0 & \\
\hline No & 86.8 & 74.2 & 88.0 & 93.0 & 0.047 \\
\hline
\end{tabular}

Values are means \pm SD

${ }^{a} p$ value for at least one of the four groups showing a statistically significant difference from the others

individuals, but only in Asian-Indians and not in Chinese. C3 and C5 acylcarnitines, previously shown to be elevated in association with the increase in BCAAs in obese vs lean individuals from the south-eastern USA [14], tended to be higher in the high-HOMA group in both the Asian-Indian and Chinese men, but this change did not achieve statistical significance (ESM Table 1).

Hormone and cytokine profiling Table 4 shows the hormonal profile of the various groups. In both ethnic groups, IR was associated with elevated plasma leptin and reduced adiponectin, but in addition leptin levels were clearly elevated in the Asian-Indians compared with the Chinese, regardless of HOMA group. IGF-I levels were negatively associated with HOMA in the Chinese, but this was no longer statistically significant after adjustment for age and BMI. More strikingly, IGF-I levels were higher in Chinese than in Asian-Indian individuals, irrespective of the degree of IR. IGFBP1 levels were sharply decreased as a function of HOMA in both ethnic groups, but overall levels were not different between Chinese and Asian-Indian individuals. Among cytokines, TNF- $\alpha$ levels were elevated in highHOMA vs low-HOMA quartiles, but these changes only reached statistical significance for Chinese individuals and were attenuated after adjustment for age and BMI.

PCA PCA was applied to all analytes measured and all study participants, and identified 26 factors grouping in biologically plausible clusters (ESM Table 3). Factor 4 was composed of the BCAAs leucine/isoleucine, as well as phenylalanine, 
Table 2 Dietary intake and physical activity

\begin{tabular}{|c|c|c|c|c|c|}
\hline \multirow[t]{2}{*}{ Variable } & \multicolumn{2}{|l|}{ Chinese } & \multicolumn{2}{|l|}{ Asian-Indian } & \multirow[t]{2}{*}{$p$ value } \\
\hline & $\begin{array}{l}\text { Low HOMA } \\
(n=97)\end{array}$ & $\begin{array}{l}\text { High HOMA } \\
(n=83)\end{array}$ & $\begin{array}{l}\text { Low HOMA } \\
(n=30)\end{array}$ & $\begin{array}{l}\text { High HOMA } \\
(n=53)\end{array}$ & \\
\hline \multicolumn{6}{|l|}{ Dietary intake } \\
\hline Total energy (kJ/day) & $9,808 \pm 4,039$ & $9,406 \pm 3,022$ & $8,816 \pm 3,922$ & $9,502 \pm 2,826$ & 0.607 \\
\hline Total fat (g/day) & $74 \pm 40$ & $69 \pm 29$ & $68 \pm 41$ & $74 \pm 23$ & 0.716 \\
\hline Total carbohydrate (g/day) & $334 \pm 127$ & $322 \pm 106$ & $298 \pm 122$ & $324 \pm 107$ & 0.552 \\
\hline Total protein (g/day) & $85 \pm 37$ & $82 \pm 29$ & $70 \pm 31$ & $73 \pm 23$ & 0.061 \\
\hline Total saturated fat (g/day) & $29 \pm 17$ & $27 \pm 12$ & $28 \pm 21$ & $31 \pm 11$ & 0.451 \\
\hline Energy from fat $(\%)$ & $27.7 \pm 5.0$ & $27.4 \pm 5.7$ & $28.3 \pm 6.4$ & $29.6 \pm 5.4$ & 0.15 \\
\hline Energy from carbohydrate (\%) & $57.5 \pm 5.6$ & $57.6 \pm 6.9$ & $57.3 \pm 5.8$ & $56.8 \pm 5.7$ & 0.868 \\
\hline Energy from protein $(\%)$ & $14.5 \pm 1.7$ & $14.7 \pm 1.7$ & $13.5 \pm 2.1$ & $12.9 \pm 1.2$ & $4.478 \times 10^{-9}$ \\
\hline Energy from saturated fatty acids (\%) & $10.8 \pm 2.6$ & $10.7 \pm 2.2$ & $11.5 \pm 3.4$ & $12.5 \pm 3.1$ & 0.001 \\
\hline Total weight of rice alternatives (g/day) & $554 . \pm 231$ & $514 \pm 169$ & $404 \pm 220$ & $465 \pm 222$ & 0.004 \\
\hline Starch (g/day) & $178 \pm 77$ & $175 \pm 62$ & $173 \pm 54$ & $163 \pm 69$ & 0.656 \\
\hline Sugar (g/day) & $122 \pm 61$ & $125 \pm 83$ & $117 \pm 61$ & $130 \pm 139$ & 0.925 \\
\hline Physical activity (kJ/week) & $3,332 \pm 4,672$ & $2,813 \pm 3,357$ & $3,901 \pm 3,621$ & $3,654 \pm 4,002$ & 0.537 \\
\hline
\end{tabular}

${ }^{a} p$ value for at least one of the four groups showing a statistically significant difference from the others

tyrosine and methionine. Although not meeting our a priori criteria for inclusion based on factor loading (factor load $\geq 0.4)$, factor 4 also included glutamate/glutamine (0.29), alanine (0.30), proline (0.22) and valine (0.22). Consistent with the results for the individual metabolites, factor 4 scores differed significantly across groups ( $p$ value for overall $F$ test 0.003 ), and were significantly higher in individuals with highHOMA compared with individuals with low HOMA, in both Chinese $(p=0.005)$ and Asian-Indians $(p=0.02)$, even after adjusting for age and BMI ( $p=0.04$ and 0.02 , respectively) (Table 5). In addition, factor 4 was significantly correlated with HOMA across all individuals $\left(r^{2}=0.18, p=0.003\right)$.

Factor 8, composed of pyruvate, lactate and arginine, was also significantly different between groups, with higher levels in high-HOMA individuals in both ethnic groups. This finding mirrored results for the individual analytes of this factor. Factor 13, composed of isobutyrylglycine and isovalerylglycine, was significantly lower in the high-HOMA vs lowHOMA group in Asian-Indians but not in Chinese, again similar to the results for individual metabolites in the factor. Factor 21, composed of IGF-I and IGFBP3 was significantly lower in high-HOMA Chinese individuals vs low-HOMA Chinese individuals, and was not significant in Asian-Indians. No other factors were significantly different between highand low-HOMA individuals.

\section{Discussion}

Using a comprehensive metabolic profiling approach, we have found a clear association between a subset of amino acids and IR in Chinese and Asian-Indian individuals living in Singapore. In a previous study that applied the same tools to a group of obese (BMI $37 \mathrm{~kg} / \mathrm{m}^{2}$ ) vs lean (BMI $23 \mathrm{~kg} / \mathrm{m}^{2}$ ) individuals residing in the southeastern USA, striking increases in amino acid levels were noted in the obese individuals, including BCAAs and several of their metabolites [14]. The current findings strongly support the idea that these changes are related to IR rather than obesity per se, since we observed changes in many of the same individual amino acids in high vs low tertiles of IR in a group of Chinese individuals with a mean BMI of approximately $24 \mathrm{~kg} / \mathrm{m}^{2}$. Although the number of AsianIndian individuals available limited the power of this study to detect associations between individual metabolites and IR in this ethnic group, BCAAs and related amino acids trended in the same direction in Asian-Indians as observed in the Chinese, and the PCA-derived factor 4 composed of BCAAs and related amino acids was associated significantly with IR in both ethnic groups.

As essential amino acids, BCAA levels in blood are controlled by dietary intake, their rate of use for the anabolic processes of protein biosynthesis and cell growth, their rate of catabolism through transamination and the branched-chain keto acid dehydrogenase complex, and the rate of protein turnover/hydrolysis. In our prior study, we reported that obese individuals consumed more protein than lean individuals [14]. In the present study, whereas dietary protein intake differed between ethnic groups, it did not differ between individuals with high or low HOMA. Possible explanations for the increased levels of BCAAs in individuals with high HOMA in the current study could 


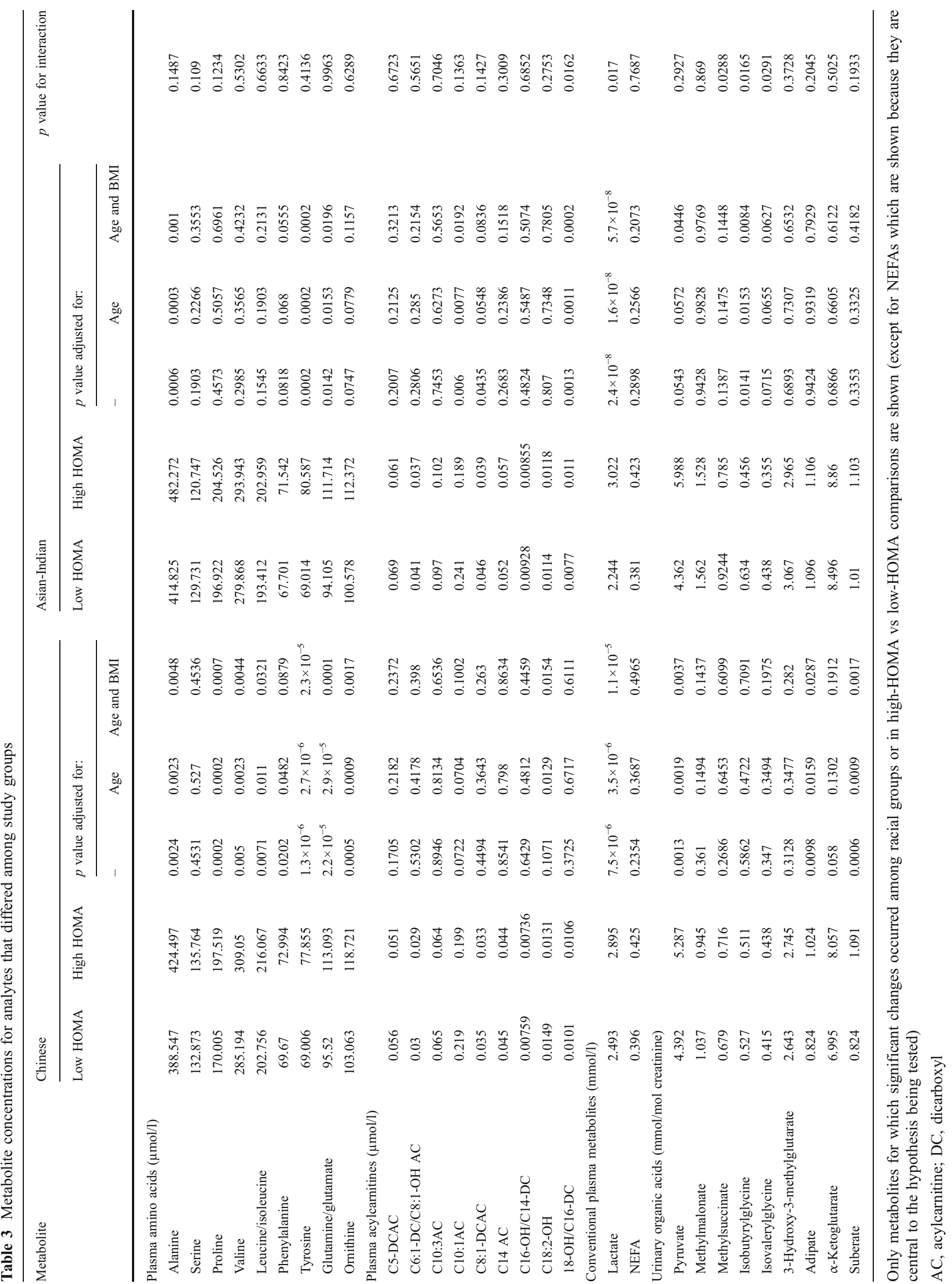


Table 4 Hormone profiles

\begin{tabular}{|c|c|c|c|c|c|c|}
\hline & \multirow[t]{2}{*}{ Low HOMA } & \multirow[t]{2}{*}{ High HOMA } & \multicolumn{3}{|c|}{$p$ value adjusted for: } & \multirow[t]{2}{*}{$p$ value for interaction } \\
\hline & & & - & Age & Age and BMI & \\
\hline \multicolumn{7}{|l|}{ Chinese } \\
\hline TNF- $\alpha(\mathrm{pg} / \mathrm{ml})$ & 4.37 & 4.84 & 0.0336 & 0.1736 & 0.1515 & 0.9964 \\
\hline Leptin (ng/ml) & 6.77 & 10.53 & $7.9 \times 10^{-11}$ & $1.6 \times 10^{-10}$ & $1.6 \times 10^{-9}$ & 0.8964 \\
\hline IGF-I (ng/ml) & 204.23 & 185.47 & 0.041 & 0.2625 & 0.5032 & 0.6478 \\
\hline IGFBP1 (pg/ml) & $23,581.28$ & $17,016.29$ & 0.0066 & 0.0002 & 0.0013 & 0.5867 \\
\hline Adiponectin (ng/ml) & $7,670.07$ & $6,338.04$ & 0.0074 & 0.0007 & 0.0056 & 0.2123 \\
\hline \multicolumn{7}{|l|}{ Asian-Indian } \\
\hline $\mathrm{TNF}-\alpha(\mathrm{pg} / \mathrm{ml})$ & 4.88 & 5.34 & 0.4999 & 0.3988 & 0.3694 & 0.9964 \\
\hline Leptin (ng/ml) & 10.29 & 14.23 & 0.0143 & 0.0147 & 0.0437 & 0.8964 \\
\hline IGF-I (ng/ml) & 173.23 & 161.48 & 0.2467 & 0.1632 & 0.1339 & 0.6478 \\
\hline IGFBP1 (pg/ml) & $24,548.88$ & $15,555.55$ & 0.023 & 0.0319 & 0.0593 & 0.5867 \\
\hline Adiponectin (ng/ml) & $8,808.63$ & $6,334.19$ & 0.0024 & 0.0043 & 0.0048 & 0.2123 \\
\hline
\end{tabular}

include increased protein turnover and/or reduced rates of BCAA catabolism. Support for the former idea comes from studies showing higher rates of leucine appearance in obese compared with lean individuals, and even higher rates of protein turnover in obese diabetic individuals compared with obese non-diabetic individuals [11]. On the other hand, a separate group reported no change in overall rate of leucine appearance in obese compared with lean individuals, and an actual decrease in appearance of leucine from forearm and adipose tissue in the obese individuals [36]. A major difference in the two studies was that individuals were studied in the post-absorptive state or after short-term fasting in the former case, and after $22 \mathrm{~h}$ of fasting in the latter case. Obesity has been reported to be associated with changes in the abundance of BCAA-catabolising enzymes in adipose tissue [37], but the contribution of these changes to plasma BCAA levels has not been demonstrated. Interestingly, in the current study, we saw increases in some metabolites reflective of BCAA catabolism (e.g. glutamate/ glutamine, alanine, $\mathrm{C} 3$ and $\mathrm{C} 5$ acylcarnitines), but decreases in others (urinary isobutyrylglycine and isovalerylglycine). We are not aware of any data on protein turnover or expression of BCAA-catabolic enzymes in individuals matched for a relatively low body weight but differing in insulin sensitivity. Thus, further work will be required to fully address the mechanisms underlying the increases in amino acid levels in insulin-resistant individuals of the type studied here.

Despite similar BMI, insulin-resistant and insulin-sensitive individuals in the current study had markedly different waist circumferences. This suggests that despite similar BMI, individuals who were insulin resistant in this study may have had increased central adiposity, which is often associated with reduced lean body mass, although neither of these variables was directly measured in the current study. Central adiposity accompanied by reduced lean body mass is a condition that has been termed 'sarcopenic obesity' [38], and its presence is consistent with the strongly elevated plasma leptin levels observed in the individuals with high HOMA from both ethnic groups in the current study. Assuming that the increased levels of select amino acids observed in insulinresistant individuals in the current study are indicative of increased protein turnover, this profile could be consistent with sarcopenia. Also consistent with this idea, IR is independently correlated with poor muscle strength and with accelerated loss of muscle strength and quality in older individuals with type 2 diabetes [39-42], although such relationships are not apparent in all studies [43, 44].

Downregulation of the growth hormone axis has also been implicated in the link between IR and sarcopenia. Levels of IGF-I were reduced in insulin-resistant individuals in the present study, similar to our prior findings in obese, insulin-resistant North American individuals [14]. This could potentially result in decreased anabolic use of amino acids, including BCAAs, for protein synthesis and cell growth, contributing to their rise in the circulation. Interestingly, Chinese individuals had higher IGF-I levels than Asian-Indian individuals, possibly contributing to the particular susceptibility of the latter group to develop metabolic diseases. We also considered the possibility that increased inflammation associated with obesity and IR may result in accelerated muscle catabolism [45, 46]. However, the levels of pro-inflammatory cytokines were only minimally elevated in our insulin-resistant individuals.

When BMI is controlled as in the current study, no correlation is evident between HOMA and either NEFA levels or levels of lipid-derived metabolites such as 


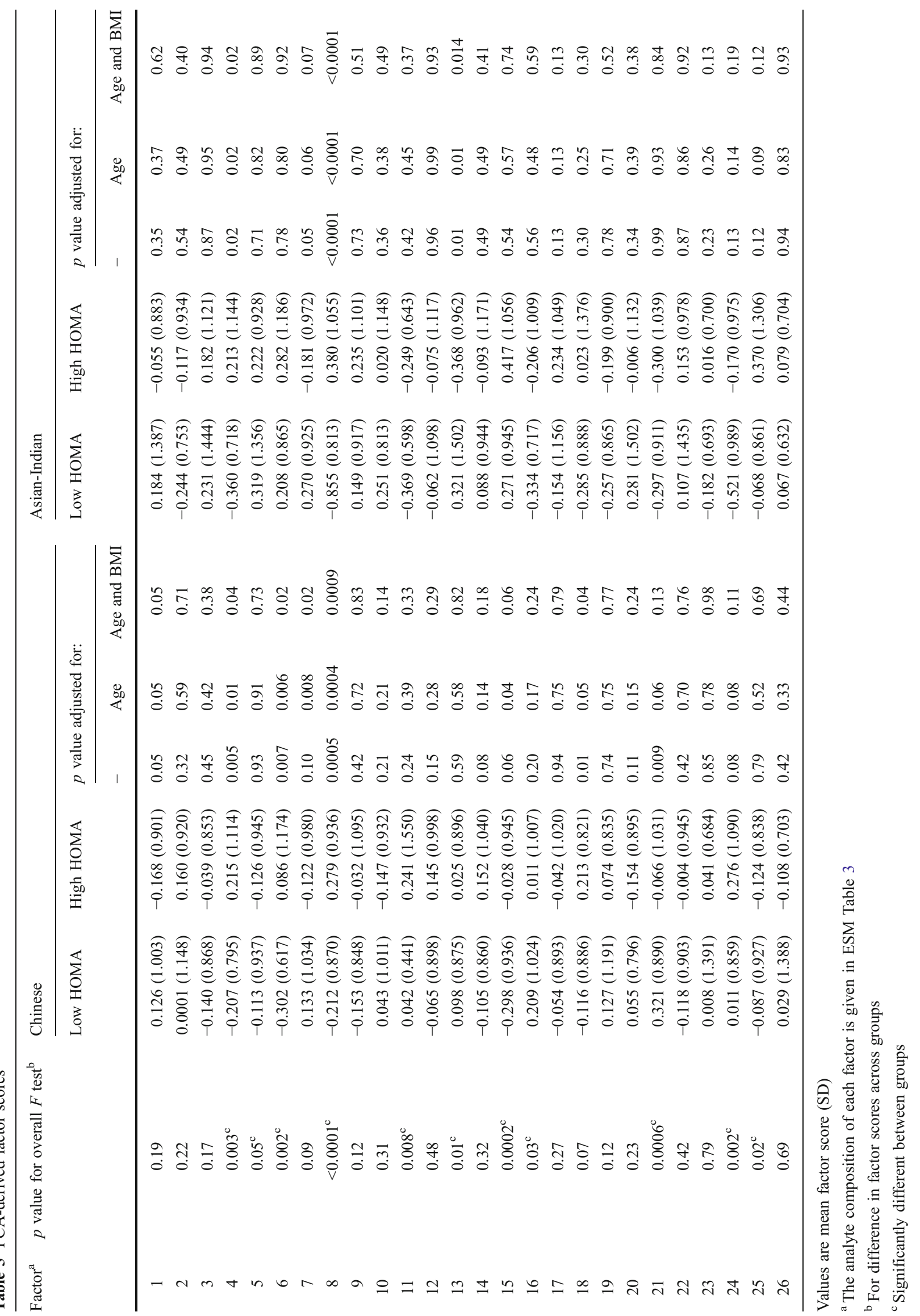


acylcarnitines or ketones. Increases in NEFAs have been reported in obese and insulin-resistant individuals in many prior studies, leading to extensive studies on the potential 'lipotoxic' effects of these agents in development of IR [3-7]. It should be noted that the absence of a rise in circulating NEFA in the insulin-resistant individuals in the current study does not mean that rates of fatty acid turnover were unchanged [47, 48], and flux measurements would be needed to fully address this point. Another striking finding of our study is the dramatic lowering of IGFBP1 in the high-HOMA individuals in both ethnic groups. Insulin is known to suppress production of IGFBP1 in the liver [49]. These findings suggest that the elevated plasma insulin in insulin-resistant individuals was largely effective for suppression of lipolysis and NEFA levels, as well as hepatic IGFBP1 production. However, these same elevated insulin levels apparently failed to control the levels of BCAAs and related amino acids. Whether this is indicative of selective IR at the level of suppression of protein turnover and amino acid catabolism or whether the reduced IGF-I levels play a more prominent and primary role is a matter that will require further investigation.

Several limitations of the study should be noted. First, we have only studied men, and the relevance of these findings to women is not known at this time. Second, we have only studied these individuals in the fasting or postabsorptive state. Third, HOMA is an imprecise method for assessing IR. However, individuals from opposing tertiles of HOMA index had clear differences in the levels of blood lipids (triacylglycerol and HDL-cholesterol) and adiponectin, supporting the idea that these individuals had true differences in insulin sensitivity.

In conclusion, significant evidence supports the role of NEFAs and inflammation in the pathogenesis of obesityassociated IR. Our study complements this knowledge, by showing that when IR develops at relatively low body weight it is no longer associated with NEFA and inflammation but instead with a metabolic signature of elevated BCAAs and related metabolites. It remains to be determined if the rise in BCAAs and related metabolites is an early manifestation of IR caused by other factors, or is actually one of the primary causes of the insulin-resistant state. Support for a primary role of amino acids includes recent studies in animals showing that feeding of BCAAs causes IR, particularly in the context of a high-fat diet [14]. On the other hand, hepatic steatosis is an example of a factor that could trigger an early state of IR [50], leading to a secondary rise in BCAAs and their metabolites. It will also be important to determine if insulin-resistant individuals such as those studied here with relatively low body weight actually do have enhanced protein catabolism and other markers of reduction of lean body mass/sarcopenia. If so, and given that increased physical activity is known to attenuate muscle loss during ageing or weight loss [51], future work might be directed at investigation of the impact of physical activity on amino acid profiles and IR in nonobese individuals. Regardless of the outcome of these additional studies, the current work has shown that BCAAs and related metabolites are associated with IR even in the absence of obesity, and suggest that dysregulation of amino acid metabolism may be an early event in the progression to more severe IR and type 2 diabetes.

Acknowledgements The studies reported in this article were supported by a Khoo Discovery Project Award from the Duke-National University of Singapore Graduate Medical School (to E. S. Tai), and by NIH grant PO1DK58398 (to C. B. Newgard). The Singapore Prospective Study Program (from which the samples used in this study were derived) was funded by the Biomedical Research Council of Singapore (grant no. 03/1/27/18/216). Samples were archived and maintained at the Singhealth Tissue repository prior to analysis.

Duality of interest The authors declare that there is no duality of interest associated with this manuscript.

\section{References}

1. Abbasi F, Brown BW Jr, Lamendola C, McLaughlin T, Reaven GM (2002) Relationship between obesity, insulin resistance, and coronary heart disease risk. J Am Coll Cardiol 40:937-943

2. Lee J, Ma S, Heng D et al (2007) Should central obesity be an optional or essential component of the metabolic syndrome? Ischemic heart disease risk in the Singapore Cardiovascular Cohort Study. Diabetes Care 30:343-347

3. Boden G, Shulman GI (2002) Free fatty acids in obesity and type 2 diabetes: defining their role in the development of insulin resistance and beta-cell dysfunction. Eur J Clin Invest 32(Suppl 3):14-23

4. Savage DB, Petersen KF, Shulman GI (2007) Disordered lipid metabolism and the pathogenesis of insulin resistance. Physiol Rev 87:507-520

5. Summers SA (2006) Ceramides in insulin resistance and lipotoxicity. Prog Lipid Res 45:42-72

6. Koves TR, Ussher JR, Noland RC et al (2008) Mitochondrial overload and incomplete fatty acid oxidation contribute to skeletal muscle insulin resistance. Cell Metab 7:45-56

7. Muoio DM, Newgard CB (2008) Mechanisms of disease: molecular and metabolic mechanisms of insulin resistance and beta-cell failure in type 2 diabetes. Nat Rev Mol Cell Biol 9:193-205

8. Schenk S, Saberi M, Olefsky JM (2008) Insulin sensitivity: modulation by nutrients and inflammation. J Clin Invest 118: 2992-3002

9. Chevalier S, Marliss EB, Morais JA, Lamarche M, Gougeon R (2005) Whole-body protein anabolic response is resistant to the action of insulin in obese women. Am J Clin Nutr 82:355-365

10. Felig P, Marliss E, Cahill GF Jr (1969) Plasma amino acid levels and insulin secretion in obesity. N Engl J Med 281:811-816

11. Gougeon R, Morais JA, Chevalier S, Pereira S, Lamarche M, Marliss EB (2008) Determinants of whole-body protein metabolism in subjects with and without type 2 diabetes. Diabetes Care 31:128-133

12. Marliss EB, Gougeon R (2002) Diabetes mellitus, lipidus et ... proteinus! Diabetes Care 25:1474-1476

13. Pereira S, Marliss EB, Morais JA, Chevalier S, Gougeon R (2008) Insulin resistance of protein metabolism in type 2 diabetes. Diabetes 57:56-63 
14. Newgard CB, An J, Bain JR et al (2009) A branched-chain amino acid-related metabolic signature that differentiates obese and lean humans and contributes to insulin resistance. Cell Metab 9:311-326

15. Huffman KM, Shah SH, Stevens RD et al (2009) Relationships between circulating metabolic intermediates and insulin action in overweight to obese, inactive men and women. Diabetes Care 32: $1678-1683$

16. Zimmet P, Alberti KG, Shaw J (2001) Global and societal implications of the diabetes epidemic. Nature 414:782-787

17. Deurenberg-Yap M, Chew SK, Lin VF, Tan BY, van Staveren WA, Deurenberg P (2001) Relationships between indices of obesity and its co-morbidities in multi-ethnic Singapore. Int J Obes Relat Metab Disord 25:1554-1562

18. Ko GT, Chan JC, Cockram CS, Woo J (1999) Prediction of hypertension, diabetes, dyslipidaemia or albuminuria using simple anthropometric indexes in Hong Kong Chinese. Int J Obes Relat Metab Disord 23:1136-1142

19. Thai AC, Yeo PP, Lun KC et al (1990) Diabetes mellitus and its chronic complications in Singapore: an increasing healthcare problem. Ann Acad Med Singapore 19:517-523

20. Cutter J, Tan BY, Chew SK (2001) Levels of cardiovascular disease risk factors in Singapore following a national intervention programme. Bull World Health Organ 79:908-915

21. Tai ES, Lim SC, Chew SK, Tan BY, Tan CE (2000) Homeostasis model assessment in a population with mixed ethnicity: the 1992 Singapore National Health Survey. Diabetes Res Clin Pract 49:159-168

22. Tan CE, Emmanuel SC, Tan BY, Jacob E (1999) Prevalence of diabetes and ethnic differences in cardiovascular risk factors. The 1992 Singapore National Health Survey. Diabetes Care 22:241-247

23. Yeo KK, Tai BC, Heng D et al (2006) Ethnicity modifies the association between diabetes mellitus and ischaemic heart disease in Chinese, Malays and Asian Indians living in Singapore. Diabetologia 49:2866-2873

24. Nang EE, Khoo CM, Tai ES et al (2009) Is there a clear threshold for fasting plasma glucose that differentiates between those with and without neuropathy and chronic kidney disease?: the Singapore Prospective Study Program. Am J Epidemiol 169:1454-1462

25. Hughes K, Yeo PP, Lun KC et al (1990) Cardiovascular diseases in Chinese, Malays, and Indians in Singapore. II. Differences in risk factor levels. J Epidemiol Community Health 44:29-35

26. Hughes K, Aw TC, Kuperan P, Choo M (1997) Central obesity, insulin resistance, syndrome $\mathrm{X}$, lipoprotein(a), and cardiovascular risk in Indians, Malays, and Chinese in Singapore. J Epidemiol Community Health 51:394-399

27. Deurenberg-Yap M, Li T, Tan WL, van Staveren WA, Chew SK, Deurenberg P (2001) Can dietary factors explain differences in serum cholesterol profiles among different ethnic groups (Chinese, Malays and Indians) in Singapore? Asia Pac J Clin Nutr 10:39-45

28. Deurenberg-Yap M, Li T, Tan WL, van Staveren WA, Deurenberg P (2000) Validation of a semiquantitative food frequency questionnaire for estimation of intakes of energy, fats and cholesterol among Singaporeans. Asia Pac J Clin Nutr 9:282-288

29. Anon (1994) Food consumption study 1993. Food and Nutrition Department, Ministry of Health, Singapore

30. Block G, Hartman AM, Dresser CM, Carroll MD, Gannon J, Gardner L (1986) A data-based approach to diet questionnaire design and testing. Am J Epidemiol 124:453-469

31. Matthews DR, Hosker JP, Rudenski AS et al (1985) Homeostasis model assessment: insulin resistance and B-cell function from fasting plasma glucose and insulin concentrations in man. Diabetologia 28:412-419

32. An J, Muoio DM, Shiota M et al (2004) Hepatic expression of malonyl-CoA decarboxylase reverses muscle, liver and wholeanimal insulin resistance. Nat Med 10:268-274
33. Ferrara CT, Wang P, Neto EC et al (2008) Genetic networks of liver metabolism revealed by integration of metabolic and transcriptional profiling. PLoS Genet 4:e1000034

34. Wu JY, Kao HJ, Li SC et al (2004) ENU mutagenesis identifies mice with mitochondrial branched-chain aminotransferase deficiency resembling human maple syrup urine disease. J Clin Invest 113:434-440

35. Shah SH, Hauser ER, Bain JR et al (2009) High heritability of metabolomic profiles in families burdened with premature cardiovascular disease. Mol Syst Biol 5:258

36. Patterson BW, Horowitz JF, Wu G et al (2001) Regional adipose tissue amino acid metabolism in lean and obese women. Am J Physiol Endocrinol Metabol 282:E931-E936

37. She P, van Horn C, Reid T et al (2007) Obesity-related elevations in plasma leucine are associated with alterations in enzymes involved in branched-chain amino acid metabolism. Am J Physiol Endocrinol Metabol 293:E1552-E1563

38. Roubenoff R (2000) Sarcopenic obesity: does muscle loss cause fat gain? Lessons from rheumatoid arthritis and osteoarthritis. Ann N Y Acad Sci 904:553-557

39. Abbatecola AM, Ferrucci L, Ceda G et al (2005) Insulin resistance and muscle strength in older persons. J Gerontol Ser A Biol Sci Med Sci 60:1278-1282

40. Guillet C, Boirie Y (2005) Insulin resistance: a contributing factor to age-related muscle mass loss? Diabetes Metab 31(Spec No. 2):5S20-5S26

41. Nomura T, Ikeda Y, Nakao S et al (2007) Muscle strength is a marker of insulin resistance in patients with type 2 diabetes: a pilot study. Endocr J 54:791-796

42. Park SW, Goodpaster BH, Strotmeyer ES et al (2007) Accelerated loss of skeletal muscle strength in older adults with type 2 diabetes: the health, aging, and body composition study. Diabetes Care 30:1507-1512

43. Kuk JL, Kilpatrick K, Davidson LE et al (2008) Whole body skeletal muscle mass is not related to glucose tolerance or insulin sensitivity in overweight and obese men and women. Appl Physiol Nutr Metab 33:769-774

44. Sparrow D, Borkan GA, Gerzof SG et al (1986) Relationship of fat distribution to glucose tolerance. Results of computed tomography in male participants of the Normative Aging Study. Diabetes 35:411-415

45. Roubenoff R, Freeman LM, Smith DE, Abad LW, Dinarello CA, Kehayias JJ (1997) Adjuvant arthritis as a model of inflammatory cachexia. Arthritis Rheum 40:534-539

46. Schrager MA, Metter EJ, Simonsick E et al (2007) Sarcopenic obesity and inflammation in the InCHIANTI Study. J Appl Physiol 102:919-925

47. Thyfault JP, Kraus RM, Hickner RC et al (2004) Impaired plasma fatty acid oxidation in extremely obese women. Am J Physiol Endorinol Metabol 287:E1076-E1081

48. Blaak EE, Wolffenbuttel BH, Saris WH et al (2001) Weight reduction and the impaired plasma-derived free fatty acid oxidation in type 2 diabetic subjects. J Clin Endocrinol Metabol 86:1638-1644

49. Brismar K, Fernqvist-Forbes E, Wahren J, Hall K (1994) Effect of insulin on the hepatic production of insulin-like growth factorbinding protein-1 (IGFBP-1), IGFBP-3, and IGF-I in insulindependent diabetes. J Clin Endocrinol Metabol 79:872-878

50. Petersen KF, Dufour S, Befroy D, Lehrke M, Hendler RE, Shulman GI (2005) Reversal of nonalcoholic hepatic steatosis, hepatic insulin resistance, and hyperglycemia by moderate weight reduction in patients with type 2 diabetes. Diabetes 54:603-608

51. Amati F, Dube JJ, Coen PM et al (2009) Physical inactivity and obesity underlie the insulin resistance of aging. Diabetes Care 32:1547-1549 